\title{
Expresión de cadherina E y cateninas en los carcinomas uroteliales.
}

\author{
San Miguel Fraile P, Gómez de María C, Donis Quintairos L, Carrera Vazquez A, \\ Iglesias Martínez P, Barreiro Barbosa $\mathrm{M}^{\mathrm{a}} \mathrm{J}$.
}

Servicio de Anatomía Patológica. Hospital POVISA. Vigo. Pontevedra.

Actas Urol Esp. 2007;31(4):355-360

\section{RESUMEN}

\section{EXPRESIÓN DE CADHERINA E Y CATENINAS EN LOS CARCINOMAS UROTELIALES}

Objetivo: Determinar la correlación entre la expresión de cadherina $\mathrm{E}$ y $\alpha, \beta$ y $\gamma$ cateninas detectadas con técnicas de inmunohistoquímica, con las variables pronósticas clásicas del carcinoma urotelial: grado histológico, estadio clínico y recurrencia.

Material y métodos: Hemos estudiado la expresión de cadherina $\mathrm{E}$ y $\alpha, \beta$ y $\gamma$ cateninas en 37 biopsias de carcinomas transicionales de vejiga. Diecinueve corresponden a carcinomas transicionales de vejiga no recurrentes y 18 a carcinomas transicionales de vejiga recurrentes. Hemos estudiado estadísticamente la asociación de estos marcadores con el estadio clínico, grado histológico y recurrencia del tumor.

Resultados: Demostramos una correlación estadísticamente significativa entre la expresión de cadherina $\mathrm{E}$ y $\gamma$ catenina con el estadio clínico del tumor. No demostramos asociación estadística significativa entre la expresión de $\alpha$ y $\beta$ catenina con el grado histológico, estadio clínico y recurrencia del tumor.

Conclusión: La expresión aberrante de cadherina E y $\gamma$ catenina parece estar relacionado con la capacidad invasiva de los carcinomas uroteliales.

Palabras claves: Carcinoma urotelial.Vejiga. Cadherina E. Cateninas.

\section{ABSTRACT \\ EXPRESION OF E-CADHERIN AND CATENINS IN UROTHELIAL CARCINOMAS}

Objetive: We examined the presence of E-cadherin and $\alpha, \beta$ and $\gamma$ catenins detected by immunohistochemistry and correlated with the classic variables: grade, stage and recurrence.

Material and method: The authors evaluated 37 transitional cell carcinomas. Biopsy specimens included no recurrent transitional cell carcinoma $(\mathrm{n}=19)$ and recurrent transitional cell carcinoma $(\mathrm{n}=18)$. Association of E-cadherin and $\alpha, \beta$ and $\gamma$ catenins immunoreactivity with tumor grade, clinical stage and tumor recurrence was examined.

Results: E-cadherin and $\gamma$ catenin expression were related to the clinical stage of the disease, $\alpha$ and $\beta$ catenins were not correlated with grade, stage and recurrence of disease.

Conclusions: Decreased E cadherin and $\gamma$ catenin expression were associated with advanced stage of the disease.

Keywords: Urothelial carcinoma. Bladder. E-cadherin. Catenin.

$\mathrm{E}^{\prime}$ carcinoma urotelial de vejiga representa aproximadamente el $2 \%$ de todos los tumores malignos ${ }^{1}$. El $80 \%$ de estos tumores son superficiales y aproximadamente entre el 30-90\% recidivan después de la extirpación y el 15-20\% de estas recurrencias son como tumores invasivos y/o metastásicos ${ }^{2-3}$. Tradicionalmente, se ha considerado que el grado histológico y el estadio clínico son los factores pronósticos más importantes en el carcinoma transicional de vejiga, 
pero en muchas ocasiones no es posible predecir con estos criterios la recurrencia y pronóstico del tumor.

Por otra parte, está ampliamente aceptado que la adhesión celular se halla frecuentemente reducida en las neoplasias, y que esta reducción de la adhesión celular se asocia a una mayor diseminación e infiltración de las células neoplásicas. Se han descrito varias familias de moléculas de adhesión celular, que incluyen las cadherinas y cateninas. Las cadherinas son moléculas dependientes de calcio, y son las principales responsables de esta función. Estas moléculas son glicoproteinas transmembrana con una porción citoplasmática que interacciona con el citoesqueleto a través de las cateninas.

Se han publicado diversos estudios sobre el valor pronóstico de la expresión de la cadherina $\mathrm{E}$ en los carcinomas transicionales de vejiga. En varios trabajos se ha demostrado que una disminución en la expresión de cadherina E está asociado a carcinomas transicionales de alto grado histológico y estadio clínico avanzado ${ }^{4-9}$, pero los resultados son controvertidos, y no esta todavía definido el significado pronóstico de la expresión de estas moléculas ${ }^{10}$. El objetivo de este estudio es valorar la expresión de cadherina $\mathrm{E}$ y cateninas en los carcinomas transicionales de vejiga y estudiar la correlación de estos con el grado histológico, estadio clínico y recurrencia.

\section{MATERIAL Y MÉTODOS}

Hemos estudiado retrospectivamente 37 biopsias de vejiga tomadas al azar del archivo de nuestro servicio que fueron diagnosticadas de carcinoma transicional de vejiga entre los años 1995-2003. Diecinueve biopsias corresponden a carcinomas transicionales de vejiga no recurrentes y dieciocho a carcinomas transicionales de vejiga recurrentes. La muestra analizada incluyó: 9 tumores en estadio pTa (26\%), 18 tumores con estadio pT1 (47\%) y 10 tumores con estadio pT2 (26\%). En los tumores recurrentes el estudio inmunohistoquímico se realizó en la primera biopsia. El período de seguimiento mínimo en los carcinomas
Tabla 1

Características de los distintos anticuerpos empleados con técnicas de inmunohistoquímica

\begin{tabular}{lcccc}
\hline Anticuerpo & Clon & Disolución & Procedencia & Tiempo de incubación \\
\hline Catenina $\alpha$ & $25 \mathrm{~B} 1$ & $1: 25$ & Novocastra & 30 minutos \\
Catenina $\beta$ & 14 & $1: 50$ & Becton Dickinson & 30 minutos \\
Catenina $\gamma$ & 15 & $1: 500$ & Becton Dickinson & 30 minutos \\
Cadherina $\mathrm{E}$ & 36 & $1: 2000$ & Becton Dickinson & 30 minutos \\
\hline
\end{tabular}

uroteliales fue de 43 meses. En todos los casos se revisaron las biopsias para confirmación del diagnóstico histológico.

Para la inmunohistoquímica se utilizaron los anticuerpos monoclonales $\alpha$ catenina, $\beta$ catenina, $\gamma$ catenina y E-cadherina, cuyo fabricante, dilución de trabajo y designación del clon viene especificado en la Tabla 1. La valoración de todos los marcadores inmunohistoquímicos se hizo cualitativamente según los criterios ya estableci$\operatorname{dos}^{11,12}$, de manera que se clasificaron en: positiva (> del $90 \%$ de las células se tiñen), focalmente positiva (cuando se tiñen < del 10\% de las células) y heterogénea (cuando se tiñen entre el 10$90 \%$ de las células). En todos los casos se especifico si la tinción era de membrana o citoplasmática. Cuando la tinción era positiva se consideró como normal y cuando la tinción era heterogénea, focalmente positiva o negativa fue considerado como expresión aberrante. (Figs. 1, 2 y 3)

La técnica de inmunohistoquímica se realizó sobre las secciones tratadas previamente a la inmunotinción para facilitar el acceso de los anticuerpos a los antígenos tisulares. Se utilizó la olla a presión durante 5 minutos en tampón citrato $\mathrm{pH}$ 6,0 . Posteriormente se realizó una inhibición de la peroxidasa endógena con peróxido de hidrógeno al $3 \%$ durante 10 minutos y se incubaron con el anticuerpo primario diluido adecuadamente en tampón tris a pH 7,2 que contiene Na N3 $15 \mathrm{mmol} / \mathrm{L}$ durante 30 minutos. El método utilizado para la detección fue el de estreptoavidina marcada con peroxidasa-biotina y el cromógeno de revelado fue diaminobencidina.

Antes de proceder a la cuantificación de los resultados de inmunohistoquímica, se valoró en todos los casos la calidad de la técnica y se seleccionaron las áreas con más positividad, evitando mediciones en áreas periféricas, zonas desvitalizadas, con necrosis o artefacto. 


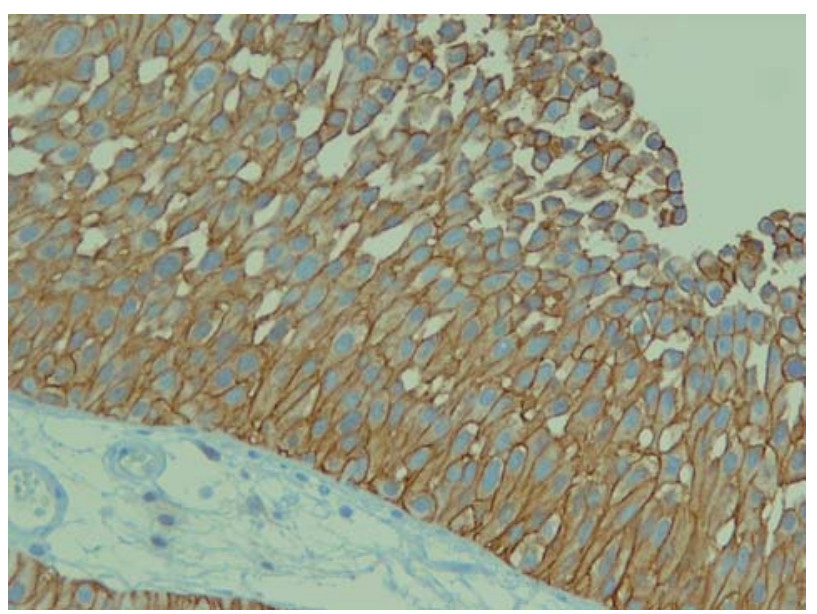

FIGURA 1: Tinción positiva (cadherina E).

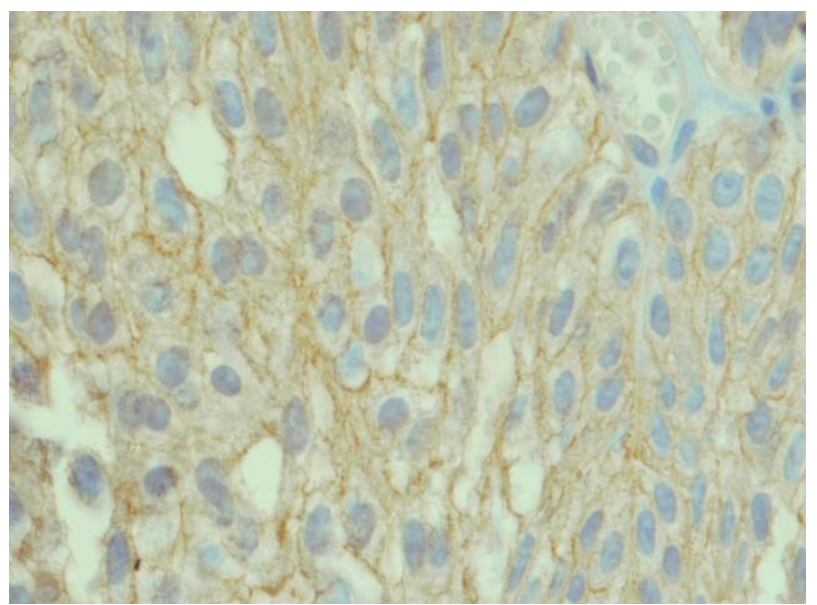

FIGURA 2: Tinción heterogénea (cadherina E).

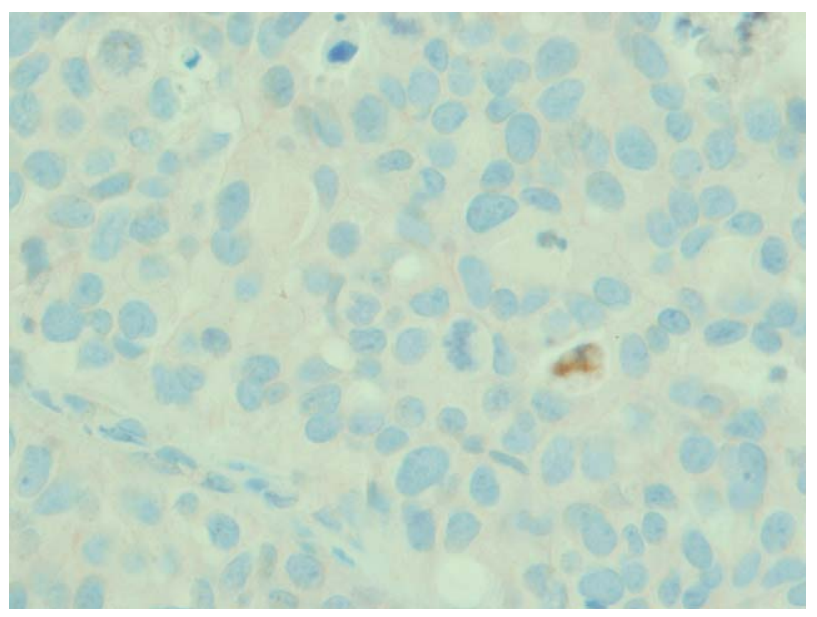

FIGURA 3: Tinción negativa (cadherina E).

En el estudio estadístico se incluyeron las siguientes variables: grado histológico (G1,G2 o G3), estadio clínico (pTa, pT1 o pT2) y recurrencia (sí o no) con la expresión de los distintos mar- cadores immunohistoquímicos: $\alpha$ catenina, $\beta$ catenina, $\gamma$ catenina y cadherina E. El estudio estadístico se analizó mediante la prueba exacta de Fisher. Se consideró que un valor era estadísticamente significativa, cuando $\mathrm{p} \leq 0.05$.

\section{RESULTADOS}

Las características clínico patológicas se muestran en la Tabla 2. La edad de los enfermos osciló entre los 34 y 89 años (media=69). El seguimiento clínico está comprendido entre los 132 y 43 meses (media 77 y 59 meses para los tumores no recurrentes y recurrentes respectivamente).

Tabla 2

Característica clínicas e histológicas de los carcinomas transicionales.

\begin{tabular}{lcc}
\hline & $\begin{array}{c}\text { Tumores } \\
\text { recurrentes } \\
(\mathbf{n = 1 8 )}\end{array}$ & $\begin{array}{c}\text { Tumores } \\
\text { no recurrentes } \\
(\mathbf{n}=\mathbf{1 9 )}\end{array}$ \\
\hline Edad Media & 68 años & 74 años \\
Sexo: & & \\
Hombre. & 14 & 17 \\
Mujer & 4 & 2 \\
Grado histológico: & & \\
G1 & 1 & 2 \\
G2 & 8 & 7 \\
G3 & 9 & 10 \\
Estadio clínico: & & \\
pTa & 4 & 5 \\
pT1 & 9 & 9 \\
pT2 & 5 & 5 \\
\hline
\end{tabular}

La expresión de los distintos marcadores inmunohistoquímicos según el grado histológico, estadio clínico y recurrencia del tumor está reflejada en las Tablas 3, 4 y 5.

El estudio estadístico entre las tres variables analizadas (grado histológico, estadio clínico y recurrencia) con los distintos parámetros inmunohistoquímicos se refleja en las Tabla 3, 4 y 5 . Se demostró asociación estadística significativa entre la expresión de $\gamma$ catenina y Ecadherina con el estadio clínico del tumor ( $\mathrm{p}=0,012$ y $\mathrm{p}=0,016)$, apreciándose un incremento de la expresión aberrante de esta catenina y cadherina conforme aumenta el estadio clínico del tumor. En el resto de los marcadores inmunohistoquímicos estudiados no se demos- 
Tabla 3

Expresión de cadherina E y cateninas según el estadio clínico

\begin{tabular}{lccc}
\hline $\begin{array}{l}\text { Estadio } \\
\text { clínico }\end{array}$ & $\begin{array}{c}\text { Tinción } \\
\text { normal } \\
\text { n (\%) }\end{array}$ & $\begin{array}{c}\text { Tinción } \\
\text { aberrante } \\
\text { n (\%) }\end{array}$ & $\begin{array}{c}\text { Valor } \\
\text { de p }\end{array}$ \\
\hline $\begin{array}{c}\text { Cadherina E: } \\
\text { pTa }\end{array}$ & $9(100)$ & $0(0)$ & $\mathbf{p}=\mathbf{0 , 0 1 6}$ \\
pT1 & $13(64)$ & $5(36)$ & \\
pT2 & $4(40)$ & $6(60)$ & \\
Catenina- $\alpha$ & & $7(88)$ & \\
pTa & $2(22)$ & $17(99)$ & \\
pT1 & $1(1)$ & $9(90)$ & \\
pT2 & $1(10)$ & & $\mathrm{p}=0,419$ \\
Catenina- $\beta$ & $9(100)$ & 0 & \\
pTa & $15(84)$ & $3(16)$ & \\
pT1 & $9(90)$ & $1(10)$ & \\
pT2 & & & $\mathbf{p}=\mathbf{0 , 0 1 2}$ \\
Catenina- $\gamma$ & $9(100)$ & 0 & \\
pTa & $18(95)$ & $1(5)$ & \\
pT1 & $7(70)$ & $3(30)$ & \\
pT2 & & & \\
\hline
\end{tabular}

Tabla 4

Expresión de cadherina E y cateninas según el grado histológico

\begin{tabular}{lccc}
\hline $\begin{array}{l}\text { Grado } \\
\text { histológico }\end{array}$ & $\begin{array}{c}\text { Tinción } \\
\text { normal } \\
\text { n (\%) }\end{array}$ & $\begin{array}{c}\text { Tinción } \\
\text { aberrante } \\
\text { n (\%) }\end{array}$ & $\begin{array}{c}\text { Valor } \\
\text { de p }\end{array}$ \\
\hline $\begin{array}{c}\text { Cadherina E: } \\
\text { G1 }\end{array}$ & $3(100)$ & $0(0)$ & $\mathrm{p}=0,254$ \\
G2 & $11(79)$ & $3(210)$ & \\
G3 & $12(60)$ & $8(40)$ & \\
Catenina- $\alpha$ & & & $\mathrm{p}=0,293$ \\
G1 & $1(33)$ & $2(77)$ & \\
G2 & $2(14)$ & $12(86)$ & \\
G3 & $1(5)$ & $19(95)$ & \\
Catenina- $\beta$ & $3(100)$ & 0 & $\mathrm{P}=0,630$ \\
G1 & $13(93)$ & $1(7)$ & \\
G2 & $16(80)$ & $4(20)$ & \\
G3 & & & $\mathrm{P}=0,250$ \\
Catenina- $\gamma$ & $3(100)$ & $2(14)$ & \\
G1 & $12(86)$ & $4(20)$ & \\
G2 & $16(84)$ & & \\
G3 & & & \\
\hline
\end{tabular}

tró asociación estadística significativa con el estadio clínico, grado histológico y recurrencia del tumor.

\section{DISCUSIÓN}

El carcinoma urotelial es el tumor maligno más frecuente de la vejiga. Histológicamente, es muy importante diferenciar los carcinomas transicionales superficiales de los tumores que infiltran músculo. Los primeros son tumores poco
Tabla 5

Expresión de cadherina E y cateninas según la recurrencia

\begin{tabular}{lccc}
\hline Recurrencia & $\begin{array}{c}\text { Tinción } \\
\text { normal } \\
\text { n (\%) }\end{array}$ & $\begin{array}{c}\text { Tinción } \\
\text { aberrante } \\
\text { n (\%) }\end{array}$ & $\begin{array}{c}\text { Valor } \\
\text { de p }\end{array}$ \\
\hline $\begin{array}{l}\text { Cadherina E: } \\
\text { Si }\end{array}$ & $11(62)$ & $7(38)$ & $\mathrm{p}=0,235$ \\
No & $15(79)$ & $4(21)$ & \\
Catenina- $\alpha$ & & & $\mathrm{p}=0,323$ \\
$\quad \mathrm{Si}$ & $1(5)$ & $17(95)$ & \\
No & $3(15)$ & $16(85)$ & \\
Catenina- $\beta$ & & & $\mathrm{p}=0,677$ \\
$\mathrm{Si}$ & $16(88)$ & $2(12)$ & \\
No & $17(90)$ & $2(10)$ & \\
Catenina- $\gamma$ & & & $\mathrm{p}=0,125$ \\
$\mathrm{Si}$ & $16(83)$ & $2(17)$ & \\
No & $16(85)$ & $3(15)$ & \\
\hline
\end{tabular}

agresivos pero con un índice alto de recurrencias, mientras que los segundos son tumores más agresivos, en general con mal pronóstico. El 80\% de los carcinomas transicionales de vejiga son superficiales y aproximadamente entre el 30-90\% recidivan después de la extirpación y el 15-20\% de las recurrencias son invasivas y/o metastási$\mathrm{cas}^{2-3}$. Sería importante poder identificar a estos pacientes con tumores potencialmente más agresivos que podrían beneficiarse de la cirugía y quimioterapia y/o radioterapia.

Tradicionalmente se ha considerado que el grado histológico y el estadio clínico son los factores pronósticos más importantes en el carcinoma transicional de vejiga, sin embargo, hay una gran variabilidad inter e intra observador, en el grado histológico de la neoplasia ${ }^{11}$. Por este motivo en los últimos años se ha intentado buscar nuevos marcadores biológicos que nos permitiesen mejorar esta capacidad pronóstica.

La integridad de los mecanismos de adhesión celular es fundamental para el mantenimiento de la arquitectura tisular. La organización de las células en el tejido epitelial sano se lleva a cabo por varias moléculas y uniones intercelulares, entre las que se encuentran el grupo de las cadherinas y cateninas. La pérdida o disminución de cadherina $\mathrm{E}$ se ha relacionado con la invasividad tumoral, alto grado histológico y estadio clínico avanzado $^{4,7,12,13}$. Sin embargo, son pocos los estudios que han valorado la expresión de $\alpha, \beta$ y $\gamma$ catenina en el carcinoma transicional de vejiga. 
Muchos estudios han verificado la relación existente entre la pérdida de expresión de la Ecadherina con la capacidad de invasión y metástasis en múltiples carcinomas, entre ellos el carcinoma urotelial ${ }^{4,7,12,13}$. Estos trabajos sugieren que la expresión aberrante de cadherina E es un marcador de progresión y agresividad tumoral ${ }^{4,7,12,13}$. En nuestra serie, el 30\% de las neoplasias mostraban expresión aberrante de cadherina E (el 20\% de los tumores superficiales y el 60\% de los tumores infiltrantes). Estos resultados son ligeramente inferiores a los aportados por Koksal et al. ${ }^{11}$, pero similares a los aportados por Bringuier et al. Por otra parte, nosotros hemos confirmado resultados similares a los observados en otros trabajos en los que se demuestra asociación estadística significativa entre la expresión anómala de cadherina $\mathrm{E}$ con el estadio clínico del tumor ${ }^{4,7,11}$. Sin embargo, en nuestro estudio no hemos detectado asociación estadística entre la expresión aberrante de cadherina E y el grado histológico del tumor. Estos resultados difieren con parte de las series publicadas ${ }^{4,8}$, pero coinciden con los aportados por otros auto$\operatorname{res}^{11,12,14,15}$. Además, al igual que otros trabajos hemos observado que la mayoría de los tumores con expresión aberrante tenían un patrón inmunohistoquímico heterogéneo con áreas positivas y negativas ${ }^{4,16}$. Finalmente, nosotros no hemos demostrado asociación entre expresión aberrante de cadherina $\mathrm{E}$ y recurrencia tumoral en tumores uroteliales de vejiga. Clairotte et al. han observado que la expresión aberrante de cadherina E no esta asociada a progresión tumoral en los carcinomas uroteliales superficiales ${ }^{16}$.

La expresión aberrante de $\alpha$ y $\beta$ cateninas en los carcinomas uroteliales de vejiga de nuestra serie fue del 89 y 11\% respectivamente; y no demostramos asociación estadística significativa con el grado histológico, estadio clínico ni recurrencia de la neoplasia. Son todavía pocas las publicaciones que han estudiado estos dos marcadores $^{9,11,16}$, pero en algunas de las series se ha demostrado asociación estadística con el estadio y grado del tumor ${ }^{11,16}$. A nuestro entender, estos resultados deben ser confirmados mediante estudios en series más amplias.

Por último, son pocos los trabajos que han estudiado el valor pronóstico de la tinción de la $\gamma$ catenina en los carcinomas uroteliales de vejiga ${ }^{7,9,16}$.
La expresión aberrante de $\gamma$ catenina en los carcinomas uroteliales de vejiga de nuestra serie fue del 18\%. Con respecto a la asociación de la expresión de la $\gamma$ catenina con el grado histológico y el estadio clínico se aprecian marcadas variaciones según las distintas series ${ }^{7,9,16}$. Nuestros resultados concuerdan con los presentados por Syringos et al. ${ }^{8}$ y Clairotte et al. ${ }^{16}$ ya que apreciamos una pérdida de la expresión normal de la $\gamma$ catenina conforme aumenta el estadio clínico del tumor. Sin embargo, no hemos apreciado que la expresión aberrante de $\gamma$ catenina guarde relación estadística significativa con el grado histológico del tumor ni con la recurrencia de la neoplasia. Clairotte et al. consideran que la expresión aberrante de $\gamma$ catenina puede ser un marcador pronóstico de progresión tumoral especialmente en los tumores en estadio $\mathrm{I}^{16}$.

En resumen, nuestros resultados sugieren que la expresión aberrante de cadherina $\mathrm{E}$ y $\gamma$ catenina podrían ser un indicador de estadio clínico avanzado en los carcinomas uroteliales de vejiga. Finalmente, hemos observado frecuentemente expresión aberrante de todos los parámetros inmunohistoquímicos valorados en este estudio por lo que muy posiblemente estén implicados en la patogénesis y evolución de la neoplasia urotelial.

\section{REFERENCIAS}

1. Heiken JP, Forman HP, A, Brown JJ. Neoplasms of the bladder, prostate and testis. Radiol Clin North Am. 1994;32(1): 81-98.

2. Heney NM, Ahmed S, Flanagan MJ, Frable W, Corder MP, Hafermann MD et al. Superficial bladder cancer: progression and recurrence. J Urol. 1983;130(6):1083-1086.

3. Soloway MS. Intravesical therapy for bladder cancer. Urol Clin North Am. 1988;15(4):661-669.

4. Bringuier PP, Umbas R, Schaafsma HE, Karthaus HF, Debruyne FM, Schalken JA. Decreased E-cadherin immunoreactivity correlates with poor survival in patients with bladder tumors. Cancer Res 1993;53(14):3241-3245.

5. Fujisawa M, Miyazaki J, Takechi Y, Arakawa S, Kamidono $\mathrm{S}$. The significance of E-cadherin in transitional-cell carcinoma of the human urinary bladder. World $J$ Urol. 1996;14 Suppl 1:S12-15.

6. Shimoyama Y, Nagafuchi A, Fujita S, Gotoh M, Takeichi M, Tsukita S, et al. Cadherin dysfunction in a human cancer cell line:possible involvement of loss of alpha-catenin expression in reduced cell-cell adhesiveness. Cancer Res. 1992;52(20):5770-5774.

7. Syrigos KN, Harrington K, Waxman J, Krausz T, Pignatelli M. Altered gamma-catenin expression correlates with poor survival in patients with bladder cancer. J Urol. 1998;160 (5): 1889- 1893. 
8. Syrigos KN, Krausz T, Waxman J, Pandha H, RowlinsonBusza $G$, Verne $J$ et al. E-cadherin expression in bladder cancer using formalin-fixed, paraffin-embedded tissues: correlation with histopathological grade, tumour stage and survival. Int J Cancer. 1995;64(6):367-370.

9. Shimazui T, Schalken JA, Giroldi LA, Jansen CF, Akaza H, Koiso $\mathrm{K}$ et al. Prognostic value of cadherin-associated molecules ( $\alpha-, \beta-$, and $\gamma$-catenins and p120 cas) in bladder tumors. Cancer Res. 1996;56(18):4154-4158.

10. Rieger-Christ KM, Cain JW, Braasch JW, Dugan JM, Silverman ML, Bouyounes B et al. Expression of classic cadherins type I in urothelial neoplastic progression. Hum Pathol. 2001;32(1):18-23.

11. Koksal IT, Ates M, Danisman A, Sezer C, Ciftcioglu A, Karpuzoglu G et al.Reduced E-cadherin and $\gamma$-catenin expressions have no prognostic role in bladder carcinoma. Pathol Oncol Res. 2006(1);12:13-19.

12. Lipponen PK, Eskelinen MJ. Reduced expression of E-cadherin is related to invasive disease and frequent recurrence in bladder cancer. J Cancer Res Clin Oncol. 1995;121 (5):303-308.

13. Nakanishi K, Kawai T, Torikata C, Aurues T, Ikeda T. Ecadherin expression in upper urinary tract carcinoma. Int J Cancer. 1997;74(4):446-449.
14. Ross JS, del Rosario AD, Figge HL, Sheehan C, Fisher HA, Bui HX. E-cadherin expression in papillary transitional cell carcinoma of the urinary bladder. Hum Pathol. 1995;26(9):940-944.

15. Byrne RR, Shariat SF, Brown R, Kattan MW, Morton RA JR, Wheeler TMet al. E-cadherin immunostaining of bladder transitional cell carcinoma, carcinoma in situ and lymph node metastases with long-term follow-up. J Urol. 2001;165(5):1473-1479.

16. Clairotte A, Lascombe I, Fauconnet S, Mauny F, Felix S, Algros MP et al. Expression of E-cadherin and alpha-, beta-, gamma-catenins in patients with bladder cancer: identification of gamma-catenin as a new prognostic marker of neoplastic progression in T1 superficial urothelial tumors. Am J Clin Pathol 2006;125(1):119-126.

Correspondencia autora: Dra. P. San Miguel Fraile. Servicio de Anatomía Patológica. Hospital Povisa Salamanca, 5. 36211 Vigo (Pontevedra).

Tel.: 986-413144 Ext: 1161.

E-mail autora: psanmiguel@povisa.es

Información artículo: Original - Cáncer de vejiga

Trabajo recibido: septiembre 2006

Trabajo aceptado: enero 2007 